\title{
Selective Excitation of the Yellow Luminescence of GaN
}

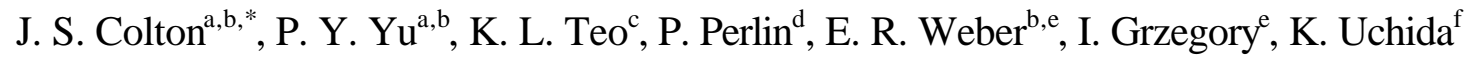 \\ ${ }^{a}$ Department of Physics, University of California, Berkeley, CA 94720 \\ ${ }^{\mathrm{b}}$ Materials Sciences Division, Lawrence Berkeley National Laboratory, Berkeley, CA 94720 \\ ${ }^{\mathrm{c}}$ Department of Electrical Engineering, National University of Singapore, Singapore (119260) \\ ${ }^{\mathrm{d}}$ UNIPRESS, High Pressure Research Center, Polish Academy of Sciences, 01-142 Warsaw, Poland \\ ${ }^{\mathrm{e}}$ Department of Materials Science and Mineral Engineering, University of California, Berkeley CA 94720 \\ ${ }_{\mathrm{f}}^{\mathrm{f}}$ Department of Communications and Systems, The University of Electro-Communications, 1-5-1 Choufugaoka, \\ Choufu, Tokyo 182, Japan
}

\begin{abstract}
The yellow luminescence of ntype GaN has been studied with selective excitation using a combination of $\mathrm{Ar}$ ion and dye lasers. Narrower structures whose peak energies follow the excitation photon energy over the width of the yellow luminescence have been observed. Unlike the yellow luminescence excited by above band gap excitations, these fine structures exhibits thermal activated quenching behavior. We propose that these fine structures are due to emission occurring at complexes of shallow donors and deep acceptors which can be resonantly excited by photons with energies below the band gap. The activation energy deduced from their intensity is that for delocalization of electrons out of the complexes. Our results therefore suggest that there is more than one recombination channel (usually assumed to be due to distant donor-acceptor pairs) to the yellow luminescence in GaN.
\end{abstract}

Keywords: gallium nitride, yellow luminescence, selective excitation, donor-acceptor pair complexes

\footnotetext{
* Corresponding author. e-mail: colton@ socrates.berkeley.edu
} 
In spite of the many technological advances in gallium nitride $(\mathrm{GaN})$, one basic question concerning the origin of the ubiquitous yellow luminescence (YL) band remains unanswered. Many competing explanations for its origin have been proposed, including transitions from shallow-donor to deep-acceptor[1,2,3,4,5,6], shallow-donor to deep-donor[7,8], and deep-donor to shallowacceptor[9], Even within these models, there are disagreements as to whether the YL originates from distant donor-acceptor pairs[10] (DAPs), or from localized DAP complexes[11].

One difficulty in studying the YL is its very large bandwidth $(\sim 0.5 \mathrm{eV})$. The technique of selective excitation (in which the incident laser is tuned to resonate with the emission) has become a well-established method to resolve fine structures within broad spectra as a result of inhomogeneous broadening. It has been applied successfully to materials, including $\mathrm{ZnSe}[12], \mathrm{CdSe}$ [13], porous $\mathrm{Si}$ [14], Ge microcrystals[15], and most recently GaInN[16]. In this paper we use an $\mathrm{Ar}^{+}$-ion laser as well as a continuously tunable dye laser to vary the exciting energy over the YL photon energies between 2.1 and $2.7 \mathrm{eV}$. We have observed several fine structures which we have attributed to emission from resonantly excited donor-acceptor complexes. This emission also differs from the "traditionally" excited YL (with incident photons having energy greater than the GaN band-gap, $\mathrm{E}_{\mathrm{i}}>\mathrm{E}_{\mathrm{g}}$ ) in its temperature dependence, by exhibiting thermal quenching at temperatures above $\sim 150 \mathrm{~K}$. Our results suggest that there is more than one channel responsible for the YL. While the commonly-observed YL using $E_{i}>E_{g}$ may involve distant donor-acceptor pair recombination, under resonant excitation the YL mainly originates from donor-acceptor complexes.

We have observed the same fine structures in selectively excited YL of three GaN samples. One is a $2.65 \mu \mathrm{m} \mathrm{GaN} \mathrm{film} \mathrm{grown} \mathrm{on} \mathrm{sapphire} \mathrm{by} \mathrm{MOCVD,} \mathrm{while} \mathrm{two} \mathrm{are} \mathrm{bulk} \mathrm{samples} \mathrm{grown} \mathrm{under}$ high pressure. The film was heavily ndoped with $\mathrm{Si}\left(\mathrm{n} \approx 5 \times 10^{18} \mathrm{~cm}^{-3}\right)$. Although the bulk samples 
were not intentionally doped, they also had high carrier concentrations $\left(\mathrm{n} \approx 5 \times 10^{19} \mathrm{~cm}^{-3}\right)$, most likely due to oxygen donors. Other details on their preparation and properties can be found elsewhere[17]. The photoluminescence (PL) spectra were excited by three lasers: a $50 \mathrm{~mW}$ HeCd laser at $325 \mathrm{~nm}$ $(3.814 \mathrm{eV})$ for above band gap excitation, an $\mathrm{Ar}^{+}$-ion laser with discrete laser lines between 2.4 and $2.7 \mathrm{eV}$, and a continuously tunable Coumarin 540 dye with output between 2.1 and $2.35 \mathrm{eV}$. The PL signal was analyzed with a SPEX double spectrometer and detected with a cooled GaAs photomultiplier tube and photon-counting system.

Figure 1 compares the above-band-gap-excited $\mathrm{PL}\left(\mathrm{E}_{\mathrm{i}}>\mathrm{E}_{\mathrm{g}}\right)$ with a resonantly-excited $\mathrm{YL}$ in a bulk sample measured at $12 \mathrm{~K}$. The smaller peak involving bandgap recombination at $3.5 \mathrm{eV}$ in the former spectra is not of interest to us. In that spectra, the broad band centered at $2.3 \mathrm{eV}$, with a linewidth of about $500 \mathrm{meV}$, is an example of the usual YL in GaN. When excited resonantly however, at least three narrower peaks, each having linewidths of $\sim 100 \mathrm{meV}$, become observable. For example, Fig. 1 shows excitation by the $2.541 \mathrm{eV} \mathrm{Ar}^{+}$-ion line. By deconvoluting the resonantly excited YL spectra into a sum of Lorentzians (the solid and broken curves shown in the inset of Fig. 2 are, respectively, an experimental spectrum along with its fit), we can track their emission energies as a function of excitation photon energy E. We find that these peaks appear to "follow" the excitation laser energy, in that their emission energies $\mathrm{E}_{\mathrm{YL}}$ are linearly dependent on $\mathrm{E}_{\mathrm{i}}$. This is shown in Figure 2(a) and 2(b). We have labeled these narrower peaks observed under resonant excitation as A, B', B, B" and C and their energy shifts from the incident photon energy (ie $\mathrm{E}_{\mathrm{i}}-\mathrm{E}_{\mathrm{YL}}$ ) are equal, respectively, to 40, 170, 200, 240 and $370 \mathrm{meV}$ (with uncertainties of $\pm 10 \mathrm{meV}$ ). The thin film sample shows additional peaks due to interference, labeled as IF in Fig. 2(b) inset, which are excluded from Fig. 2(b). The IF peaks can be easily distinguished from the YL peaks by noting that their energies do not change with $\mathrm{E}_{\mathrm{i}}$. The 
results from all three samples essentially agree within experimental uncertainties. The exceptions are that the finer structures B' and B" are not as clearly resolved in one bulk sample as in the other. Also, in the bulk samples peak $A$ has a slightly larger $E_{i}-E_{Y L}$ of $50 \pm 10 \mathrm{meV}$. Otherwise the similarities in these results in GaN samples grown by two completely different methods is a strong indication that similar defects are involved in their resonantly excited emission.

The selectively-excited YL differs markedly from the $\mathrm{E}_{\mathrm{i}}>\mathrm{E}_{\mathrm{g}}$ excited $\mathrm{YL}$ in its temperature dependence. One distinctive feature of the $\mathrm{E}_{\mathrm{i}}>\mathrm{E}_{\mathrm{g}}$ YL noted in previous publications has been its lack of a dependence on temperature[10,18]. Figure 3 shows the selectively-excited YL spectra $\left(E_{i}=2.471\right.$ $\mathrm{eV}$ ) of a bulk sample measured for several temperatures (T) plotted on the same intensity scale. A large thermal quenching effect is evident for $\mathrm{T}$ above $\sim 150 \mathrm{~K}$. In addition to thermal quenching, all the peaks exhibit significant broadening so that fine structures are more difficult to discern at room temperature. The inset of Fig. 3 show an Arrhenius plot of the integrated intensities I of the three peaks A, B and C. The experimental points have been fit with the following formula:

$$
I=\frac{I_{0}}{1+B \cdot e^{-E_{A} / k_{B} T}},
$$

with three adjustable parameters: $I_{o}, E_{A}$, and $B$. This equation has been derived by assuming a simple 2level rate model:

$$
\frac{d N_{2}}{d t}=G-\frac{N_{2}}{\tau_{\text {rad }}}-\frac{N_{2}}{\tau_{\text {nonrad }}},
$$

where $N_{2}$ is the population of the upper state (assumed to be proportional to the intensity I), $G$ is the excitation rate, and $\tau_{\text {rad }}$ and $\tau_{\text {nonrad }}$ are the radiative and non-radiative times for relaxing back into the lower (ground) state, respectively. Equation 1 is obtained as the steady state solution (i.e. $\mathrm{dN}_{2} / \mathrm{dT}=0$ ) 
if we assume that $\mathrm{G}$ and $\tau_{\text {rad }}$ are not dependent on temperature and $\tau_{\text {nonrad }}$ has an activated temperature dependence, given by:

$$
\frac{1}{\tau_{\text {nonrad }}}=A e^{-E_{A} / k_{B} T}
$$

Here, $E_{A}$ is the activation energy for an electron in level $\mathrm{N}_{2}$ to recombine non-radiatively and $A$ is the non-radiative recombination rate. Thus, $I_{0}=G \times \tau_{\text {rad }}$ and $B=A \times \tau_{\text {rad }}$ (i.e., $\mathrm{B}=$ the ratio of nonradiative to radiative recombination efficiency). By fitting the experimental points in Fig. 3 with Eq. (1), we obtained for peaks $\mathrm{A}, \mathrm{B}$, and $\mathrm{C}$ the activation energies of 67,57 , and $73 \mathrm{meV}$ (with uncertainties of $\pm 10 \mathrm{meV}$ ) respectively.

So far, several groups have presented evidence that the YL is due to recombination of shallow donors with deep acceptors, most likely isolated Ga vacancies. Based on this model we find it difficult to explain at least two aspects of our selectively-excited YL: (1) given the high donor concentrations in our samples (above the Mott critical density), there should be large amount of free electrons present which could recombine with the photo-excited deep acceptors to produce $\mathrm{YL}$ as broad as in the $\mathrm{E}_{\mathrm{i}}>$ $\mathrm{E}_{\mathrm{g}}$ case, and (2) the temperature dependence of the selectively-excited YL should be no different than that of above-gap excitation. In the following paragraphs we shall elaborate on these two points further.

The luminescence spectra of distant donor-acceptor pairs or DAPs (i.e., isolated donors and isolated acceptors not forming complexes) is known to be inhomogeneously broadened due to the dependence on emission energy on pair separation distance[19]. Selective excitation of ZnSe[12] and CdSe[13] has shown that narrow structures can be observed from DAP by removing the inhomogeneous broadening via resonant excitation of DAPs separated by a specific distance, determined by the excitation photon energy. However, in degenerate n-type samples, such as the GaN 
used in our study, the donors will form an impurity band and there will always be free electrons and neutral donors which can recombine with all photo-excited holes on the acceptors. As a result, if the YL is produced only by distant DAPs, the emission process would not necessarily involve the same donors as in the excitation process, and there would be no narrowing of the YL under selective excitation. In order to explain our results we therefore propose an alternate model in which radiative recombination of the resonantly excited YL takes place only at donor-acceptor complexes. It has already been suggested by both theoretical[3,20] and experimental[11,21] groups that the YL of $\mathrm{GaN}$ originates from complexes, or clusters, of shallow donor and deep acceptor pairs. However, within this model of donor-acceptor complexes, it is still necessary to assume that there is a large inhomogeneous broadening, since we are able to selectively excite the complexes over a large range of energies. At this point it is not clear as to its origin of this large broadening. One can speculate that this may arise from variations in the distance or arrangement between the donor and acceptor within the complexes, or from variations in their surroundings. Further work is necessary to determine the microscopic structure of these complexes.

We find that the fine structures in the selectively excited YL actually still have rather large widths of $\sim 0.1 \mathrm{eV}$. These large widths presumably result from homogeneous broadening. The origin of this broadening at low temperatures is most likely from the strong electron-phonon interaction which is often present in strongly localized defects. In fact, we attribute the peak A found in the resonantly excited YL to a phonon side-band of the donor-acceptor complex recombination. The origin of narrower peaks in the resonantly excited YL can in general be explained by (a) relaxation of photo-excited electrons and/or holes within the complex by emission of a phonon or (b) the complex being photo-excited into a higher energy electronic state, followed by relaxation into the ground state before emission. The former 
process most likely accounts for peak A since its energy shift of around $40 \mathrm{meV}$ lies within the range of vibrational frequencies of $\mathrm{GaN}$. On the other hand, the energy shifts of over $200 \mathrm{meV}$ for peaks $\mathrm{B}$ and $\mathrm{C}$ are too high for one phonon modes. However, these energies are plausible for excited electronic states associated with a deep acceptor. We, therefore, tentatively attributed B and C to excited electronic states of the donor-acceptor complexes.

Nevertheless, our results must be reconciled with those of YL excited by $E_{i}>E_{g}$. Recently the YL in GaN has been found to exhibit a "blue-shift" with excitation intensity[10]. These and other experiments have suggested that YL is due to recombination between spatially separated donors and acceptors. It is also necessary to develop a model which explains the different temperature dependence between the selectively-excited and above-band-gap excited YL. To account for all these experimental results, we propose that there may be multiple recombination channels which can contribute to the YL in GaN. In addition to recombination between the donor-acceptor complexes, there must also be recombination involving spatially separated or distant donors and acceptors pairs. In Table 1 we have listed a few other possible channels in addition to the two already mentioned. Whether a single channel is dominant will depend on many factors, such as: is the YL selectively-excited (which tends to favor DAP complexes), or excited by above-band-gap radiation (distant DAP pair recombination becomes possible), or excited strongly by high power density light (not only distant DAP recombination will be possible but also free-to-bound and inter-complex recombination can occur). The possible recombination channels depends also on the sample doping and temperature. A highly doped sample will contain more isolated donors and hence tend to favor distant DAP recombination. On the other hand, extended defects may attract more DAP complexes leading to stronger recombination at 
complexes. In fact, one group has reported a correlation between the strength of YL and dislocation density leading to their conclusion that the YL involves DAP complexes[11].

The existence of more than one recombination channel responsible for the YL is a key to understand why its thermal properties depend on the excitation method. For example, when a highly ndoped GaN sample is strongly excited by photons above the band-gap, the large concentration of free electrons generated tends to favor recombination via distant DAPs or even free-to-bound transitions. This recombination channel is not expected to be strongly dependent on temperature. On the other hand, during resonant excitation only a small number of DAP complexes will be selectively excited. Electrons can then be thermally excited out of these complexes. Once delocalized, these electrons will have higher probabilities of being annihilated non-radiatively. This process can lead to strong thermal quenching. Within our model, the measured activation energies of around 57-73 meV represent, therefore, the depth of traps localizing the electron in the DAP complexes. The holes are expected to be bound much more strongly to the deep acceptors.

In conclusion, we have been able to resolve fine structures in selectively-excited YL of GaN samples. Unlike the YL excited by above band-gap radiation, the selectively-excited YL exhibits strong thermal quenching. Our results suggest that selectively-excited YL involves recombination at donoracceptor pair complexes, while the YL excited by above band-gap radiation is dominated in most cases by recombination of distant donor and acceptor pairs.

\section{Acknowledgments}

The work at Berkeley was supported by the Director, Office of Energy Research, Office of Basic Energy Sciences, Materials Sciences Division, of the US Department of Energy under Contract No. DE-AC03-76SF00098. 


\section{Table 1}

\begin{tabular}{|l|c|}
\hline \multicolumn{1}{|c|}{ Possible YL Recombination Channel } & Name of Channel \\
\hline 1. donor (complex) - acceptor (same complex) & DAP complex \\
\hline 2. donor (complex) - acceptor (different complex) & inter-complex \\
\hline 3. donor (isolated) - acceptor (isolated) & distant DAP \\
\hline 4. free electron - acceptor (complex or isolated) & free-to-bound \\
\hline 5. donor (isolated) - acceptor (complex) & "hybrid complex" \\
\hline 6. donor (complex) - acceptor (isolated) & "hybrid complex" \\
\hline
\end{tabular}

\section{Table Caption}

Table 1: Various possible recombination channels contributing to the $\mathrm{YL}$ in $\mathrm{GaN}$. 


\section{Figure Captions}

FIG. 1. Comparison of the $12 \mathrm{~K}$ yellow luminescence of a bulk GaN sample between above-gap excitation (solid curve) and selective excitation (broken curve).

FIG. 2. Summary of the emission peak energies observed in the $12 \mathrm{~K}$ photoluminescence spectra of (a) bulk GaN sample and (b) GaN film, when excited by below the band gap photons of energies varying between 2.2 and $2.7 \mathrm{eV}$. Inset: examples of typical below band gap PL spectra in both bulk and thin film GaN, in this case excited at $2.471 \mathrm{eV}$. The broken curves represent a deconvolution of the experimental (solid curve) spectrum into a sum of Lorentzians. The peaks labeled "IF" in the inset of (b) are caused by interference fringes and are not plotted in the peak energy summary. The energies of peaks $\mathrm{A}, \mathrm{B}$, and $\mathrm{C}$, are 40-50, 200, and $370 \mathrm{meV}$ below the laser line, respectively.

FIG. 3. Photoluminescence of selectively-excited bulk GaN sample for various temperatures between $20 \mathrm{~K}$ (top spectrum) and $250 \mathrm{~K}$ (bottom spectrum). Inset: Arrhenius plot of the intensities of the peaks A, B, and C. By fitting the experimental points to Eq. (1) (broken curves), activation energies of 65, 57, and $73 \mathrm{meV}( \pm 10 \mathrm{meV})$ are obtained for peaks $\mathrm{A}, \mathrm{B}$, and $\mathrm{C}$, respectively. 


\section{References}

[1] T. Ogino and M. Aoki, Jpn. J. Appl. Phys. 19, 2395 (1980).

[2] P. Perlin, T. Suski, H. Teisseyre, M. Leszczynski, I. Grzegory, J. Jun, S. Porowski, P. Boguslawski,

J. Bernholc, J. C. Chervin, A. Polian, and T. D. Moustakas, Phys. Rev. Lett. 75, 296 (1995).

[3] J. Neugebauer and C. G. Van de Walle, Appl. Phys. Lett. 69, 503 (1996).

[4] H. M. Chen, Y. F. Chen, M. C. Lee, and M. S. Feng, Phys. Rev. B 56, 6942 (1997).

[5] K. Saarinen, T. Laine, S. Kuisma, J. Nissilä, P. Hautojärvi, L. Dobrzynski, J. M. Baranowski, K.

Pakula, R. Stepniewski, M. Wojdak, A. Wysmolek, T. Suski, M. Leszczynski, I. Grzegory, and S.

Porowski, Phys. Rev. Lett. 79, 3030 (1997).

[6] W. G. Perry, M. B. Bremser, and R. F. Davis, J. Appl. Phys. 83, 469 (1998).

[7] D. M. Hofmann, D. Kovalev, G. Steude, B. K. Meyer, A. Hoffmann, L. Eckey, R. Heitz, T.

Detchprom, H. Amano, and I. Akasaki, Phys. Rev. B 52, 16702 (1995).

[8] C. V. Reddy, K. Balakrishnan, H. Okumura, and S. Yoshida, Appl. Phys. Lett. 73, 244 (1998).

[9] E. R. Glaser, T. A. Kennedy, K. Doverspike, L. B. Rowland, D. K. Gaskill, J. A. Freitas, Jr., M.

Asif Khan, D. T. Olson, J. N. Kuznia, D. K. Wickenden, Phys Rev B 51, 13326 (1995).

[10] U. Kaufmann, M. Kunzer, H. Obloh, M. Maier, Ch. Manz, A. Ramakrishnan, and B. Santic, Phys

Rev B 59, 5561 (1999).

[11] G. Li, S. J. Chua, S. J. Xu, W. Wang, P. Li, B. Beaumont, and P. Gibart, Appl. Phys. Lett. 74, 2821 (1999).

[12] H. Tews, H. Venghaus, and P. J. Dean, Phys. Rev. B 23, 4097 (1981).

[13] P. Y. Yu and C. Hermann, Phys. Rev. B 23, 4097 (1981). 
[14] M. Rosenbauer, S. Finkbeiner, E. Bustarret, J. Weber, and M. Stutzmann, Phys. Rev. B 51, 10539 (1995).

[15] A. Saito and T. Suemoto, Phys. Rev. B 56, R1688 (1997))

[16] N. Wieser, O. Ambacher, H-P Felsl, L Görgens, and M. Stutzmann, APL 74, 3981 (1999)

[17] S. Porowski and I. Grzegory, in GaN and related materials, edited by J. Pearton, Optoelectronic Properties of Semiconductors and Superlattices, vol. 2, p. 295 (Gordon and Breach Science Publishers, Amsterdam, 1997).

[18] R. Zhang and T. F. Kuech, Appl. Phys. Lett. 72, 1611 (1998).

[19] P. Y. Yu and M. Cardona, Fundamentals of Semiconductors, Springer-Verlag: Berlin (1996), pg. 344 .

[20] T. Mattila and R. M. Nieminen, Phys. Rev. B 55, 9571 (1997).

[21] F. A. Ponce, D. P. Bour, W. Götz, and P. J. Wright, Appl. Phys. Lett. 68, 57 (1996). 